\title{
LETTER
}

doi:10.1017/\$1041610215001313

\section{The role of psychological well-being in obese and overweight older adults}

Being obese or overweight is often associated with impaired quality of life and psychological wellbeing (PWB) in comparison with normal-weight people (Giuli et al., 2014), both in developed and developing countries. PWB is considered a very important correlate of subjective well-being in people with excess weight. The concept of PWB is based on Ryff's multidimensional model (Ryff, 2014), which considers well-being as eudaemonic concept, and includes six dimensions: autonomy, environmental mastery, personal growth, positive relations with others, purpose in life, and selfacceptance. Few studies have analyzed the role of specific correlates of perceived well-being in the obese and overweight Italian older population. The purpose of this study was to evaluate the role of perceived well-being in obese and overweight older adults. Our study included 124 overweight and obese older participants, aged 60 years or more, selected from patients attending the Division of Endocrinology, Department of Clinical and Molecular Sciences of Polytechnic University of Marche (Italy). As previously described (Giuli et al., 2014), the participants were recruited on the basis of specific inclusion/exclusion criteria, in a period of three years (January 2010December 2012). Exclusion criteria included: (1) pituitary, thyroid, adrenal, and gonadal disorders not controlled by ongoing therapy; (2) electrolyte disorders; (3) poorly controlled diabetes mellitus (glycated haemoglobin $>8 \%$ ). All participants were clinically assessed and completed a protocol of questionnaires, including: the Hospital Anxiety and Depression Scale (HADS) used to assess levels of anxiety and depression; the PWB Scales of Ryff used to assess the six dimension that constitute the wellbeing construct; the Health-Related Quality of Life Scale (Short Form 36-item Health Survey) used to assess quality of life; the Lubben Social Network Scale used to assess the social networks and informal social support; the Psychosocial Index (PSI) used for assessing psychological distress. The included participants were mainly women $(72 \%)$ and had a mean age of 68.4 years $(S D=6.0)$. Sixty-four percentage of the participants reported fair or poor health. A correlation analysis (SPSS 16.0; data not shown) was carried out among the PWB-subscales and psychosocial variables, to select the ones to be included in the multiple linear regression models. Separated models were computed including each PWB-subscale as dependent variable. The analysis (see Table S1, available as supplementary material attached to the electronic version of this paper at www.journals.cambridge.org/jid_IPG) showed that Social support was a positive and significant predictor in four out of six models, with an important effect in PWB positive relations with others. Inversely, HADS Depression was negatively associated with PWB autonomy, PWB personal growth, and PWB self-acceptance. These results show the important role of psychological and social conditions in overweight and obese older participants, evaluated with PWB. We found an association between depression and some dimensions of PWB. The study by Risch et al. reported a similar result, because these authors suggested that a low level of PWB could represent a risk factor for depression (Risch et al., 2013). Moreover, our results highlight the importance of social support in PWB. In line with those findings, previous studies documented that older persons are more vulnerable to the effects of social isolation (Giuli et al., 2012), which predicts morbidity and mortality related to obesity and other causes. Moreover, it seems that social isolation and perception of loneliness can contribute to a higher risk of disability (Giuli et al., 2012). This highlights the importance of social support as a relevant public health issue (Dickens et al., 2011). Our results also suggest that the cognitive-behavioral interventions for enhancing perceived well-being are necessary, in particular for the prevention of depressive symptoms (Risch et al., 2013). A biopsychological perspective should be considered a valuable component in the treatment of older people affected by endocrine diseases. In particular, the use of the Ryff's PWB model could assist with the aim of gaining or maintaining a good health status in this particular clinical population. In conclusion, we suggest that the identification of predictors for PWB could be crucial when developing interventions to improve well-being and social support for overweight and obese older people.

\section{Conflict of interest}

None. 


\section{Description of authors' roles}

C.G., R.B. designed the study and drafted the article. R.P. dealt with statistical analysis. M.B., E.F., G.T. recruited participants. F.M. and F.L. supervised the protocol. All Authors critically reviewed the paper and dealt with data interpretation.

\section{Acknowledgment}

No external funding, apart from the support of the authors' institution, was available for this article.

\section{Supplementary material}

To view supplementary material for this article, please visit http://dx.doi.org/10.1017/ S1041610215001313.

\section{References}

Dickens, A. P., Richards, S. H., Greaves, C. J. and Campbel1, J. L. (2011). Interventions targeting social isolation in older people: a systematic review. BMC Public Health, 11, 647. doi: 10.1186/1471-2458-11-647.

Giuli, C., Spazzafumo, L., Sirolla, C., Abbatecola, A. M., Lattanzio, F. and Postacchini, D. (2012). Social isolation risk factors in older hospitalized individuals. Arch Gerontol Geriatr, 55, 580-585. doi:

10.1016/j.archger.2012.01.011.
Giuli, C. et al. (2014). Correlates of perceived health related quality of life in obese, overweight and normal weight older adults: an observational study. BMC Public Health, 14, 35. doi: 10.1186/1471-2458-14-35.

Risch, A. K., Taeger, S., Brüdern, J. and Stangier, U. (2013). Psychological well-being in remitted patients with recurrent depression. Psychother Psychosom, 82, 404-405. doi: 10.1159/000351808.

Ryff, C. D. (2014). Psychological well-being revisited: advances in the science and practice of eudaimonia. Psychother Psychosom, 83, 10-28. doi: 10.1159/000353263.

Cinzia Giuli, ${ }^{1}$ Roberta Papa, ${ }^{2}$

FiORELLA MARCELLINI, ${ }^{3}$ Marco Boscaro, ${ }^{4,5}$ Emanuela Faloia, ${ }^{5}$ FABRIZIA LATTANZIO, ${ }^{3}$ GIACOMO TIRABASSI ${ }^{5}$ AND ROBERTA BEVILACQUA ${ }^{3}$

${ }^{1}$ Unit of Geriatrics, INRCA (Italian National Institute on Aging), Fermo, Italy

${ }^{2}$ Centre of Socio-economic Gerontological Research, Scientific-Technological Area, INRCA (Italian

National Institute on Aging), Ancona, Italy

${ }^{3}$ Scientific Direction, INRCA (Italian National Institute on Aging), Ancona, Italy

${ }^{4}$ Endocrinology Unit, Department of Medicine, University of Padua, Padua, Italy

${ }^{5}$ Division of Endocrinology, Department of Clinical and Molecular Sciences, Umberto I Hospital, Polytechnic University of Marche, Ancona, Italy Email: c.giuli@inrca.it 